\title{
Caustics, catastrophes, and symmetries in curved beams
}

\author{
Pablo Vaveliuk, ${ }^{1, *}$ Alberto Lencina, ${ }^{1,2}$ José A. Rodrigo, ${ }^{3}$ and Oscar Martinez Matos ${ }^{3}$ \\ ${ }^{1}$ Centro de Investigaciones Ópticas (CONICET La Plata-CIC), Cno. Centenario y 506, P.O. Box 3, 1897 Gonnet, Argentina \\ ${ }^{2}$ Departamento de Física, Fac. de Cs. Exactas, Universidad Nacional de La Plata, P.O. Box 67, 1900 La Plata, \\ Pcia. de Buenos Aires, Argentina \\ ${ }^{3}$ Departamento de Óptica, Facultad de Ciencias Físicas, Universidad Complutense de Madrid, Av. Complutense s/n 28040 Madrid, Spain
}

(Received 7 April 2015; revised manuscript received 20 June 2015; published 28 September 2015)

\begin{abstract}
In this paper, a meaningful classification of optical caustic beams in two dimensions is presented. It is demonstrated that the phase symmetry of the beam's angular spectrum governs the optical catastrophe, which describes the wave properties of ray singularities, for cusp (symmetric phase) and fold (antisymmetric phase) caustics. In contrast to the established idea, the caustic classification arises from the phase symmetry rather than from the phase power, thus breaking the commonly accepted concept that fold and cusp caustics are related to the Airy and Pearcey functions, respectively. Nevertheless, the role played by the spectral phase power is to control the degree of caustic curvature. These findings provide straightforward engineering of caustic beams by addressing the spectral phase into a spatial light modulator or glass plate.
\end{abstract}

DOI: 10.1103/PhysRevA.92.033850

\section{INTRODUCTION}

Optical caustics are naturally present in everyday life and can be observed with a naked eye. They are as familiar as rainbows, bright structure in a cup of coffee illuminated by slant sunlight, and many others [1-3]. Optical caustics can be tackled from two complementary aspects: geometric and ondulatory. In the geometric approach, caustics are surfaces (in three-dimensional space) and curves (in two-dimensional space) where light rays are focused; i.e., they are concerned with envelopes of families of rays. In the ondulatory approach, caustics are regions where an electromagnetic field changes from an oscillatory behavior in the lit region, due to a strong interference effect, to an exponential decay in the shadow region, due to an evanescent field [1-3]. The stable caustics in physics present an isomorphic relationship to the elementary topological structures of catastrophe theory, being classified in the pioneering works of Whitney [4], Arnold [5], and Thom [6]. In optics, this connection is concerned with stable singularities of gradient maps of ray surfaces [1-3,7-9]. In a two-dimensional space, the standard classification allows two type of caustics: "fold" and "cusp." The former is the simplest caustics, corresponding to smooth curves and characterized by a cubic power in its generating function. Its caustic field is given by the Airy function [1-3]. In contrast, cusp catastrophes are fold caustics meeting on opposite sides of a common tangent in a cusp point. They are generated by a quartic power, and the associated caustic field is given by the Pearcey function [1-3].

Since the observation of the first curved caustic beam, the Airy beam, in 2007 [10,11], the basic and applied research on caustic laser beams grew quickly, not only in its original rectangular coordinate frame [12-15], but also in a cylindrical frame with the appearance of the circular Airy beam [16-18]. A significant advance had a place through the design of curved beams propagating along arbitrary convex trajectories $[13,14]$. All of these beams were properly tied to a fold catastrophe.

*pablov@ ciop.unlp.edu.ar
PACS number(s): 42.15.Dp, 02.40.Xx, 42.60.By, 42.60.Jf

Thereby, a question comes forth: What is the common intrinsic feature of these curved beams that leads to a fold catastrophe? Reference [13] pointed out an universal intensity pattern given by the Airy function for all of them. However, those generated in Ref. [14] have different width of the intensity lobes and different periodicity of the fringes. This means that, even if the beams in both references have qualitative similarities, the intensity profile of beams in Ref. [14] (with the exception of the Airy beam) cannot be adjusted, strictly speaking, by the Airy function. As a matter of fact, a fundamental property explaining why all these beams present a fold-type caustic is missing such that the above posed question has not been yet answered.

On the other hand, the cusp catastrophe is also allowed in two dimensions $[1-3,19]$. Its caustic field was tied to the Pearcey function having a quartic power in the generating function $[1-3,19,20]$. In spite of this, a beam exhibiting a cusplike structure was recently generated from a phase having a cubic power but with an even symmetry [21-24]. It is worth noting that the even symmetry is the common feature between the quartic phase and the absolute value of the cubic phase. Thereby, a second key question comes forth: Is any even-symmetric phase a generatrix of a cusp caustic field irrespective the value of its power?

In this paper, a meaningful classification of curved beams in two dimensions, based on symmetry considerations, is demonstrated. We assert that the common feature of fold (cusp) caustic beams lies in the odd (even) symmetry of the generating function germ of the optical catastrophe. This breaks with the universality of the cubic (quartic) power, or any other homeomorphically equivalent, as the prime generatrix of fold (cusp) caustics fields. The caustic-symmetry correspondence is rigorously demonstrated within both complementary frames, the geometric as well as the ondulatory frame. The findings of this work could be useful in the application of the catastrophe theory to other areas beyond the optical beams since the fold and cusp catastrophes are a powerful tool in diverse areas of chemistry [25,26], physics [27-29], and cosmology [30,31], in addition to other sciences such as medicine $[32,33]$. 


\section{THEORY OF CAUSTICS AND SYMMETRIES}

\section{A. Primary hypotheses}

Let us consider a $(1+1)$-dimensional wave field $E=$ $E_{0} u e^{i(2 \pi / \lambda) z}$ with a field amplitude $E_{0}$ and wavelength $\lambda$ which propagates along the $z$ axis. The dimensionless wave function $u$, expressed as a function of normalized spatial coordinates $s=x / x_{0}$ and $\xi=z \lambda /\left(2 \pi x_{0}^{2}\right)$, where $x_{0}$ is a transverse scale characterizing the beam, fulfills the paraxial wave equation: $2 i \partial_{\xi} u+\partial_{s s} u=0$. In an integral representation, $u$ can be written in terms of the angular spectrum $U(K)$ as

$$
\begin{aligned}
u(s, \xi)= & \frac{1}{2 \pi} \int_{-\infty}^{+\infty} U(K) \exp \left[i \xi\left(k^{2}-\frac{K^{2}}{2}\right)\right] \\
& \times \exp (i K s) d K,
\end{aligned}
$$

where $K$ is the dimensionless spatial frequency that is the conjugate variable of $s$, and $k=(2 \pi / \lambda) x_{0}$ is the normalized wave number. The angular spectrum allows for the experimental beam generation in a direct fashion: Once $U$ is implemented in a spatial light modulator (SLM), its Fourier transform performed by a lens creates the beam $u(s, \xi)$ in the direct coordinate space. Thereby, $U(K)$ can be viewed as the "beam's signature" since it defines the beam structure and its dynamics in propagation [21].

Our aim is to analyze the generation processes of caustics structures from the features of the angular spectrum. For this, it is assumed that the angular spectrum comprises a Gaussian amplitude and a monotonic phase with a well-defined symmetry. Without loss of generality and in order to facilitate the analytical calculations, we assume an $n$-power spectrum having a $q$ symmetry in the form

$$
U_{n}^{(q)}(K)=\exp \left(-a K^{2}\right) \exp \left[i C_{n} \operatorname{sgn}^{q}(K)|K|^{n}\right],
$$

where $\operatorname{sgn}(\cdot)$ represents the sign function and $|\cdot|$ represents the modulus operation. The Gaussian-modulated amplitude (for $a>0$ ) guarantees the square integrability of the wave function $u$. The power $n>2$ is a real number and $q$ governs the symmetry of the spectral phase: $q=0$ (or any even number) leads to an even parity, while $q=1$ (or any odd number) leads to an odd parity, irrespective of the value of the power $n$. The normalization constant $C_{n}$ guarantees the same range of phase variation for a given range of $K$.

\section{B. Geometry of caustics from symmetry considerations}

The topological and geometric features of caustics fields are obtained from the phase of the so-called catastrophe integral [1-3,7-9]. Within the angular spectrum formalism, such an integral is given by (1) whose overall phase is

$$
\Phi_{n}^{(q)}(K ; s, \xi)=C_{n} \operatorname{sgn}^{q}(K)|K|^{n}+\xi\left(k^{2}-\frac{K^{2}}{2}\right)+s K .
$$

In the language of catastrophe theory, $\Phi_{n}^{(q)}$ is called of generating function in which the spatial frequency, $K$, plays the role of internal or state variable and the spatial variables, $(s, \xi)$, act as external or control parameters [1,2]. The generating function is linear in terms of the control parameters and composed of three terms. The first one, which corresponds to the spectral phase, is the germ of the generating function, and it does not depend on the control parameters. The other two unfolding terms, depending on control variables, are the paraxial propagator term and the Fourier transform term. The ray pattern associated to the beam, and thereby its caustic structure, can be obtained by differentiating the generating function with respect to the internal variable and setting the derivative to zero [2],

$$
\frac{\partial \Phi_{n}^{(q)}}{\partial K}=n C_{n} \operatorname{sgn}^{q+1}(K)|K|^{n-1}-\xi K+s=0 .
$$

Equation (4) evaluates the stationary points, e.g., $K_{s t}$, so that each one represents a particular family of rays with linear trajectory in the $s \xi$ plane. The number of solutions $K_{s t}$ of (4) then coincides with the number of rays intersecting at a given point $(s, \xi)$. The watermark of catastrophe optics lies in that several rays can pass through the same point, which allows them to have an "envelope," which is precisely the caustic curve. On a caustic, two or more rays acquire the same direction becoming parallel [1]. In practice, a "ray annihilation" occurs on the caustics with a jumpwise variation of the number of rays passing through the caustic curve giving rise to a catastrophe.

The equations representing the caustic curves are obtained by setting to zero the second order partial derivatives of $\Phi_{n}^{(q)}$ :

$$
\frac{\partial^{2} \Phi_{n}^{(q)}}{\partial K^{2}}=n(n-1) C_{n} \operatorname{sgn}^{q}(K)|K|^{n-2}-\xi=0 .
$$

From a geometric viewpoint, Eq. (5) indicates that the projection of the ray surface onto the $s \xi$ space gives rise to singularities or catastrophes identified as caustics. Thereby, in order to obtain the equation of the caustic curve, the value of $K$, obtained from (5) must be replaced in (4). This leads to a few cases to be analyzed depending on the sign of $K$ :

Case 1: For $K \geqslant 0$, the results are identical for $q=0$ and $q=1$. From Eqs. (4)-(5),

$$
\begin{aligned}
& n C_{n} K^{n-1}-\xi K+s=0, \\
& n(n-1) C_{n} K^{n-2}-\xi=0 .
\end{aligned}
$$

Equation (7) is satisfied if, and only if, $\xi \geqslant 0$ since $n>2$ and $C_{n}>0$. Replacing $K$ from (7) into (6) and after straightforward algebra, it obtains

$$
s+A_{n} \xi^{p}=0, \quad s, \xi \geqslant 0,
$$

where the power of the caustic curve, $p$, is a function of the power of the spectral phase with explicit dependence given by

$$
p(n)=\frac{n-1}{n-2},
$$

with $p>1$. The caustic curvature coefficient, $A_{n}$, appearing in Eq. (8) is expressed as

$$
A_{n}=\frac{C_{n}^{1 /(2-n)}(2-n) n^{1 /(2-n)}}{(n-1)^{p}} .
$$

Since $A_{n}<0$, it must necessarily be $s \geqslant 0$. 
Case 2: For $K<0$ and $q=1$. From the definition of the modulus function, $|K|=-K$. From Eqs. (4)-(5),

$$
\begin{aligned}
& n C_{n}(-K)^{n-1}+\xi(-K)+s=0, \\
& -n(n-1) C_{n}(-K)^{n-2}-\xi=0 .
\end{aligned}
$$

Equation (12) is satisfied if, and only if, $\xi<0$. Replacing $(-K)$ from (12) into (11), it obtains

$$
s+A_{n}(-\xi)^{p}=0, \quad \xi<0, s \geqslant 0 .
$$

Since $A_{n}<0$ and $\xi<0$, it must necessarily be $s \geqslant 0$.

Case 3: For $K<0$ and $q=0$. From the definition of the modulus function, $|K|=-K$. From Eqs. (4)-(5),

$$
\begin{gathered}
-n C_{n}(-K)^{n-1}+\xi(-K)+s=0, \\
n(n-1) C_{n}(-K)^{n-2}-\xi=0 .
\end{gathered}
$$

Equation (15) fulfills if, and only if, $\xi \geqslant 0$. Replacing $(-K)$ from (15) into (14), it obtains

$$
A_{n} \xi^{p}-s=0, \quad \xi \geqslant 0, s<0 .
$$

Since $A_{n}<0$ and $\xi \geqslant 0$, it must necessarily be $s<0$.

\section{Fold caustics}

From the above cases, we are able to construct the caustic curves in two dimensions from symmetry considerations. We begin analyzing the odd symmetry $q=1$ : This is done from Cases 1 and 2 covering the overall spectral range $K \gtrless 0$. By combining Eqs. (8) and (13), one obtains

$$
s+A_{n}|\xi|^{p}=0 \quad s \geqslant 0 ; \forall \xi \quad \leftrightarrow \quad \text { FOLD CAUSTIC. }
$$

Equation (17) represents a convex curve since $p>1$. This is symmetric with respect to the $s$ axis, and, in addition, it is forbidden for negative values of $s$. The caustics represented by Eq. (17) are the black thick curves displayed in Fig. 1, second row, pointing out that they represent fold-type catastrophes for all the different values of the parameter $p$. The first key conclusion is: An antisymmetric spectral phase is tied to a fold catastrophe.

\section{Cusp caustics}

For the even symmetry $q=0$, Cases 1 and 3 must be analyzed. The combination of Eqs. (8) and (16) covers $K \gtrless 0$ to obtain

$$
|s|+A_{n} \xi^{p}=0 \quad \forall s ; \quad \xi \geqslant 0 \leftrightarrow \text { CUSP CAUSTIC. }
$$

Equation (18) represents two semi-infinite convex curves, one for $s>0$ and the other for $s<0$. Both curves are defined for $\xi \geqslant 0$, and they are symmetric with respect to the $\xi$ axis. In addition, they meet on the common point $(0,0)$, the cusp point, where their tangent values are infinite. These cusp-type catastrophes are depicted by thick black lines in Fig. 1, first row, for different values of the parameter $p$. The second key conclusion is: A symmetric spectral phase is tied to a cusp catastrophe.

\section{Caustics in a two-dimensional space}

Finally, Eqs. (17) and (18) can be combined in a generalized equation accounting for the symmetry of the spectral phase:

$$
\operatorname{sgn}^{q}(s)|s|+A_{n} \operatorname{sgn}^{q+1}(\xi)|\xi|^{p}=0 .
$$

Equation (19) represents the caustic curve in the $s \xi$ plane having spectral phase symmetry $q$ and real power $p$. The type of the curve is fully governed by the parameter $q$ irrespective of the value of the parameter $p$, which accounts only for its degree of curvature. The analysis performed here breaks the established idea that fold curves are necessarily related to the Airy function, and that cusp caustics are necessarily related to the Pearcey function: Any value of $n$ in the angular spectrum can give rise either to a fold curve or to a cusp curve. The key lies in the symmetry of its phase.

\section{Experimental generation guidelines}

The expressions (9)-(10) are very useful for generating curved beams with pre-engineered trajectories from a given preprogrammed phase in an SLM. The angular spectrum allows for the experimental beam generation in a direct fashion: The phase of $U(K)$ [see Eq. (2)] is implemented in an SLM that modulates an input Gaussian laser beam, which is then Fourier transformed by a lens creating the caustic beam $u(s, \xi)$ in the direct coordinate space (at the lens focal plane) as an output. Note that the SLM is illuminated by a scalar-monochromatic Gaussian laser beam with an amplitude width value $a$ in accordance with Eq. (2). Hence, one can obtain the desired power-law caustic by only implementing the proper power-law phase in the SLM. Note that the order of the caustic convexity $p$ is a decreasing function of $n$. Higher caustic curvatures occur to subcubic orders of $n$ in the limit $n \rightarrow 2$. These results are in agreement with the results derived in Ref. [18] confirming the universal relationship between the spectral phase and the beam curvature.

\section{GEOMETRIC AND ONDULATORY PICTURES}

\section{A. Geometry of rays and caustics}

Figure 1 depicts the ray patterns for caustic fields possessing symmetry $q$ and spectral power $n$. Each ray is represented by a straight line following Eq. (4) in the $s \xi$ plane. Notice that the ray slope is inversely proportional to $K_{s t}$, thus defining the direction of such a ray. Red and blue rays correspond to positive and negative values of $K_{s t}$, respectively. From the figure, the ray density increases according as the spectral power $n$ decreases (or $p$ increases). Note that a symmetric spectral phase $(q=0)$ yields three intersecting rays in each point $(s, \xi)$ within the cusp region, whereas there exists only one ray outside of this region in each point. In addition, the cusp caustic curve (18) is just the envelope of rays within the cusp region. On the other hand, an antisymmetric spectral phase $(q=1)$ leads to the fold caustic: Every point $(s, \xi)$ is crossed by two different rays in the lit region, or it does not receive any ray in the shadow region. The limit between both regions is precisely the fold caustic curve (17), that is, the envelope of rays within the fold region. The concept of "catastrophe" can also be understood from Fig. 1. The catastrophe occurs in the caustic curve as a variation of the state of the system, i.e., the 

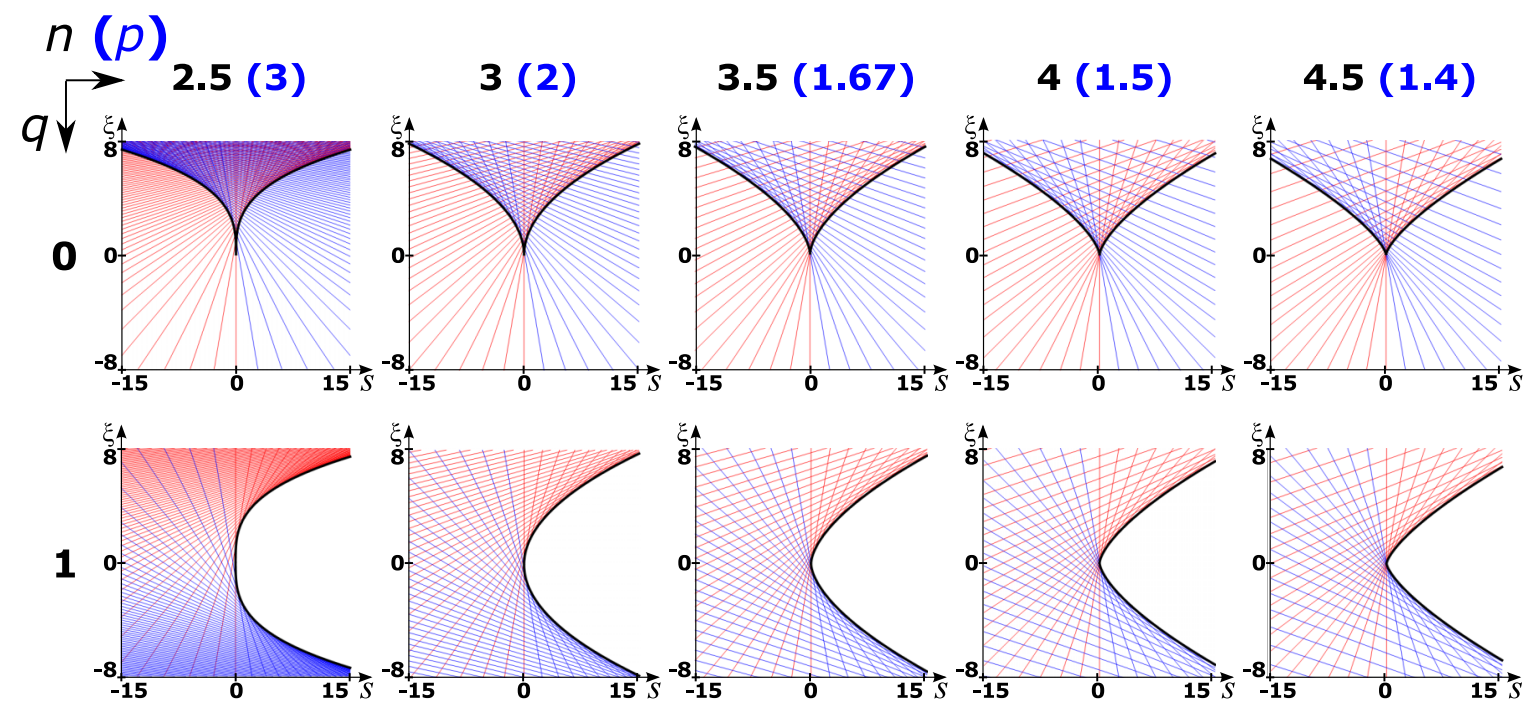

FIG. 1. (Color online) First row: Ray pattern from a symmetric spectral phase $(q=0)$ yielding a cusp caustic. Second row: Ray pattern from an antisymmetric spectral phase $(q=1)$ yielding a fold caustic. The $s$ axis is ranged in $[-15,15]$, and the $\xi$ axis is ranged in [-8,8]. The columns corresponds to different values of $n$. Red and blue rays correspond to positive and negative values of $K_{s t}$ in Eq. (4), respectively. The difference between caustics of the same kind (equal $q$ ) lies in a different degree of curvature that is given by the power $n$ (or $p$ ). The relationship between $n$ and $p$ is through Eq. (9).

number of rays intersecting at a given point $(s, \xi)$. It is clear that the type of caustics depends only on the phase symmetry. Its power $n$ produces only a different degree of curvature for the caustics.

\section{B. Geometric versus ondulatory picture}

As a final point, we will demonstrate the complementarity between the geometrical and the ondulatory pictures. The agreement between both pictures means that the constructive interference of rays inside the caustic region must necessarily match the maxima of the light intensity distribution for all values of $q$ and $n$. This comparison will validate the caustic origin of these curved beams and the novel classification in terms of the spectral phase symmetry. With this aim, the intensity distribution was derived from the numerical solution of Eq. (1). Figure 2 shows the spatial distribution of the intensity of an Airy $(n=3)$ and a Pearcey beam $(n=4)$ generated from the symmetric $(q=0)$ and the antisymmetric $(q=1)$ spectral phases. Next, we compare these intensity distributions with the pattern resulting from the constructive interference produced by pair of rays. For the fold caustic $(q=1)$, the constructive interference is achieved when

$$
\Phi_{n}^{(q=1)}\left(K_{1}^{\mathrm{f}}\right)-\left[\Phi_{n}^{(q=1)}\left(K_{2}^{\mathrm{f}}\right)-\frac{\pi}{2}\right]=2 m \pi,
$$

where $K_{1}^{\mathrm{f}}$ and $K_{2}^{\mathrm{f}}$ are two real frequencies (roots) in the caustic region, solutions of Eq. (4), and $m$ is an integer. Note that in Eq. (20) the phase corresponding to $K_{2}^{\mathrm{f}}$ has an additional phase shift of $-\pi / 2$. We analytically demonstrated this phase shift by using the method of stationary phase to find a solution for the wave function $u$ given as series expansion. Indeed, it consists in expanding the phase Eq. (3) in a Taylor series around the points $K$ in which the phase varies slowly, i.e., around the stationary points $K_{j}^{\mathrm{f}}$. The wave function $u=\sum u_{j}$ is found by expanding the phase up to second order for each $K_{j}^{\dagger}$ and solving Eq. (1):

$$
u_{j}=\frac{\exp \left[-a\left(K_{j}^{\mathrm{f}}\right)^{2}\right]}{\sqrt{2 \pi\left|\partial_{K K} \Phi_{n}^{(q)}\left(K_{j}^{\mathrm{f}}\right)\right|}} \exp \left[i \Phi_{n}^{(q)}\left(K_{j}^{\mathrm{f}}\right)+i \mu \frac{\pi}{4}\right],
$$

where $\mu$ is the sign of the second derivative of the phase. Thus, the constructive interference condition for a case with
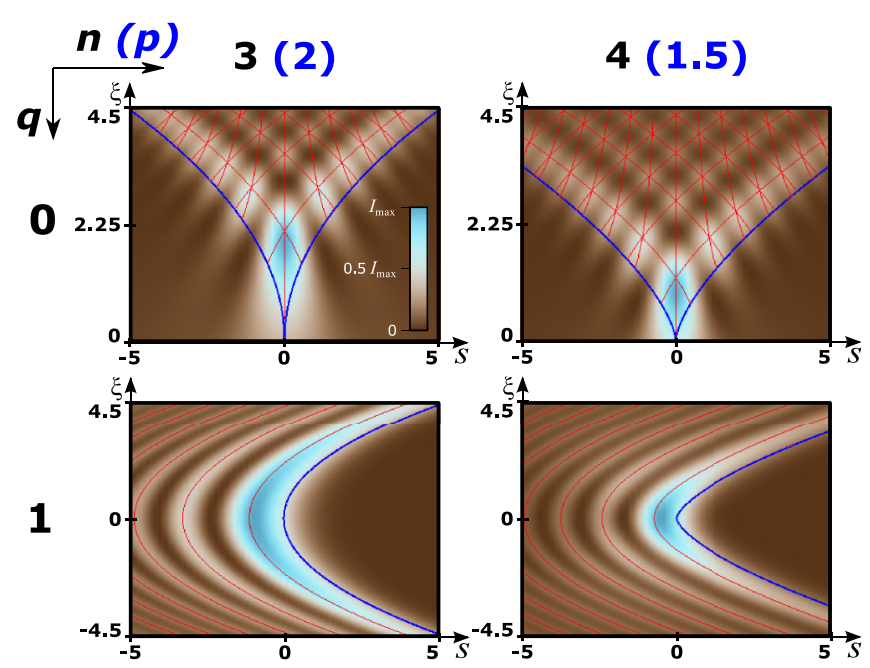

FIG. 2. (Color online) Intensity distribution for Airy $(n=3)$ and Pearcey $(n=4)$ beams, having either an even symmetry $(q=0)$ or an odd symmetry $(q=1)$, as a function of $(\xi, s)$. The $s$ axis is ranged in $[-5,5]$ for all cases, while the $\xi$ axis is ranged in $[0,4.5]$ for $q=0$ and in $[-4.5,4.5]$ for $q=1$. The intensity is scaled to $\left[0, I_{\max }\right]$. The blue curves represent the caustics. The continuum red curves indicate the constructive ray interference that are solutions of Eqs. (20) and (23). 
two stationary points is

$$
\Phi_{n}^{(q)}\left(K_{1}^{\mathrm{f}}\right)-\Phi_{n}^{(q)}\left(K_{2}^{\mathrm{f}}\right)=2\left(m-\frac{\mu_{1}-\mu_{2}}{8}\right) \pi .
$$

For the fold caustic $\mu_{1}=-\mu_{2}=1$ holds and then Eq. (20) is obtained. In the ray picture, this additional phase shift of $-\pi / 2$ was formalized by Refs. [34] and [35] and is related to the fact that the ray associated to $K_{2}^{f}$ has already "touched" the caustic while the other ray associated to $K_{1}^{f}$ not yet [2]. The curves given by Eq. (20) are represented by red curves in Fig. 2, second row. If this phase shift is not considered, the maxima of the intensity patterns do not match to the curves arising from the constructive interference of ray pairs [15].

On the other hand, the ray interference analysis for the cusp caustic beam $(q=0)$ is harder to be tackled since Eq. (4) necessarily has three real roots in the caustic region, $K_{1}^{c}, K_{2}^{c}$ and $K_{3}^{c}$. From Fig. 1 , it is clear that, at a given point $(s, \xi)$ within the caustic zone, one ray has already touched the caustic while the others two rays not yet. In this context the constructive interference is achieved through pairs of rays:

$$
\begin{array}{r}
\Phi_{n}^{(q=0)}\left(K_{1}^{\mathrm{c}}\right)-\Phi_{n}^{(q=0)}\left(K_{2}^{\mathrm{c}}\right)=2 m_{1} \pi, \\
\Phi_{n}^{(q=0)}\left(K_{1}^{\mathrm{c}}\right)-\left(\Phi_{n}^{(q=0)}\left(K_{3}^{\mathrm{c}}\right)-\frac{\pi}{2}\right)=2 m_{2} \pi, \\
\Phi_{n}^{(q=0)}\left(K_{2}^{\mathrm{c}}\right)-\left(\Phi_{n}^{(q=0)}\left(K_{3}^{\mathrm{c}}\right)-\frac{\pi}{2}\right)=2 m_{3} \pi,
\end{array}
$$

where $m_{1}, m_{2}$, and $m_{3}$ are integers and the ray that has touched the caustic curve is the associated to the root $K_{3}^{\mathrm{c}}$. Hence, the caustic phase shift only happens for Eqs. (23b) and $(23 \mathrm{c})$. The procedure to demonstrate the additional phase shift for the cusp case is an analog to the developed above by considering pairs of rays: one having touched the caustic and the other not yet. Each one of Eqs. (23) gives rise to a family of curves (red curves in Fig. 2, first row) representing the constructive interference of pairs of rays. The intersection points of them properly match the peaks of intensity of the wave field as Fig. 2 for $q=0$ shows. These results unify the geometric and ondulatory pictures by having into account the caustic classification based on the symmetries of the spectral phases.

\section{PROSPECTIVE REMARKS}

We recall that fold and cusp caustics can be experimentally created by addressing the phase $C_{n} \operatorname{sgn}(K)^{q}|K|^{n}$ of Eq. (3) onto a free-aberration SLM display. In presence of aberrations up to second order in $K$, corresponding to tilt and defocus which respectively shift the beam in the transverse and axial direction, the type of caustic is preserved. Therefore the caustic classification arises only from the symmetry of the phase addressed onto the SLM, irrespectively of such aberrations. The analysis of caustics under higher aberration orders requires further research out of the scope of this work.

The findings of this paper allow us to assume that symmetry also governs the caustic beams classification in higher dimensions (spatial, spatiotemporal, etc.). In the threedimensional case, the two-dimensional caustic curve becomes a caustic surface and the usual classification adds three new caustics: swallowtail, elliptic umbilic, and hyperbolic umbilic. However, the extension from the two-dimensional case does not seem to be something trivial. The even and odd symmetry are not more unequivocally defined since they must be referred to two canonical axes. This could lead to several symmetry variants which are out of the scope of the present work.

\section{v. CONCLUSIONS}

In this work it is demonstrated that the symmetry and the power of the beam's angular spectrum define the type of caustic and the degree of caustic curvature, respectively. No more parameters are needed for a closed-form classification of fold and cusp caustic beams. In the geometrical picture, it is found the analytical expressions for the caustics that describe the curve created by the rays' envelope. Moreover, it is proved that the number of rays intersecting the caustic at both sides varies in accordance with the catastrophe theory. In the ondulatory picture the caustic is defined by a strong interference effect due to the constructive interference of the fields associated to each family of rays. Those complementary pictures explain the symmetry dependence on the caustic type reinforcing the validity of the developed classification. Based upon these findings one is able to design unusual caustic curved beams such us a cusp-Airy beam (generated from a spectral cubic phase) and a fold-Pearcey beam (generated by a spectral quartic phase) commonly linked to fold and cusp caustics, correspondingly. This classification provides a new insight for the creation of caustics beams, which is straightforward by addressing the spectral phase into an SLM. It opens up new perspectives for the design of tailored focused beams.

\section{ACKNOWLEDGMENT}

Project TEC2014-57394-P (Spain) and CNPq 311741/2014-2 (Brazil) are acknowledged.

[1] M. V. Berry and C. Upstill, in Progress in Optics XVIII, edited by E. Wolf (North-Holland, New York, 1989).

[2] Yu. A. Kravtsov and Yu. I. Orlov, Caustics, Catastrophes and Wave Fields, 2nd ed. (Springer-Verlag, Berlin, 1999).

[3] J. F. Nye, Natural Focusing and Fine Structure of Light Caustics and Wave Dislocations (Taylor \& Francis, Philadelphia, 1999).

[4] H. Whitney, Ann. Math. 62, 374 (1955).

[5] V. I. Arnol'd, Usp. Mat. Nauk 23(1), 3 (1968); 29(2), 11 (1974); 30(5), 3 (1975).
[6] R. Thom, Structural Stability and Morphogenesis (Benjamin, New York, 1972).

[7] E. C. Zeeman, Sci. Am. 234, 65 (1976).

[8] R. Gilmore, Encyclopedia of Applied Physics (Wiley-VCH, New York, 2007).

[9] V. I. Arnold, Catastrophe Theory, 3rd ed. (Springer-Verlag, Berlin, 1992).

[10] G. A. Siviloglou and D. N. Christodoulides, Opt. Lett. 32, 979 (2007). 
[11] G. A. Siviloglou, J. Broky, A. Dogariu, and D. N. Christodoulides, Phys. Rev. Lett. 99, 213901 (2007).

[12] Y. Kaganovsky and E. Heyman, Opt. Express 18, 8440 (2010).

[13] E. Greenfield, M. Segev, W. Walasik, and O. Raz, Phys. Rev. Lett. 106, 213902 (2011).

[14] L. Froehly, F. Courvoisier, A. Mathis, M. Jacquot, L. Furfaro, R. Giust, P. A. Lacourt, and J. M. Dudley, Opt. Express 19, 16455 (2011).

[15] C. J. Zapata-Rodríguez, D. Pastor, and J. J. Miret, Opt. Express 20, 23553 (2012).

[16] I. D. Chremmos, N. K. Efremidis, and D. N. Christodoulides, Opt. Lett. 36, 1890 (2011).

[17] D. G. Papazoglou, N. K. Efremidis, D. N. Christodoulides, and S. Tzortzakis, Opt. Lett. 36, 1842 (2011).

[18] I. D. Chremmos, Z. Chen, D. N. Christodoulides, and N. K. Efremidis, Phys. Rev. A 85, 023828 (2012).

[19] M. V. Berry and S. Klein, Proc. Natl. Acad. Sci. USA 93, 2614 (1996).

[20] J. D. Ring, J. Lindberg, A. Mourka, M. Mazilu, K. Dholakia, and M. R. Dennis, Opt. Express 20, 18955 (2012).

[21] P. Vaveliuk, A. Lencina, J. A. Rodrigo, and O. Martinez Matos, Opt. Lett. 39, 2370 (2014).

[22] P. A. Quinto-Su and R. Jáuregui, Opt. Express 22, 12283 (2014).
[23] P. A. Quinto-Su and R. Jáuregui, J. Opt. Soc. Am. A 31, 2484 (2014).

[24] P. Vaveliuk, A. Lencina, J. A. Rodrigo, and O. Martinez Matos, J. Opt. Soc. Am. A 32, 443 (2015).

[25] X. Krokidis, S. Noury, and B. Silvi, J. Phys. Chem. A 101, 7277 (1997).

[26] S. Berski, Juan Andrés, B. Silvi, and L. R. Domingo, J. Phys. Chem. A 107, 6014 (2003).

[27] N. Migranov, A. Kudreyko, and D. Kondratyev, Phys. Res. Int. 2014, 1 (2014).

[28] J. G. Kim, J. W. Lee, D. C. Ihm, H. J. Lee, and E. K. Lee, J. Phys. B 36, 2267 (2003).

[29] S. Rosenblum, O. Bechler, I. Shomroni, R. Kaner, T. ArusiParpar, O. Raz, and B. Dayan, Phys. Rev. Lett. 112, 120403 (2014).

[30] N. Gouda and T. Nakamura, Prog. Theor. Phys. 81, 633 (1989).

[31] N. Gouda, Prog. Theor. Phys. 99, 55 (1998).

[32] M. N. M. Kaki, Gen. Math. Notes 11(2), 35 (2012).

[33] I. N. Stewart and P. L. Peregoy, Psych. Bull. 94, 336 (1983).

[34] V. P. Maslov, Theoria vozmushchenii i asimptoticheskie metody (Theory of Perturbations and Asymptotic Methods) (Izd. MGU, 1965) [French trans.: V. P. Maslov, Theorie des perturbations et methode asymptotique (Duod, 1972)].

[35] R. M. Lewis, Arch. Ration. Mech. Anal. 20, 191 (1965). 